\title{
Nominally forbidden transitions in the interband optical spectrum of quantum dots
}

\author{
Gustavo A. Narvaez and Alex Zunger \\ National Renewable Energy Laboratory, Golden, Colorado 80401
}

(Dated: July 18, 2006)

\begin{abstract}
We calculate the excitonic optical absorption spectra of (In,Ga)As/GaAs self-assembled quantum dots by adopting an atomistic pseudopotential approach to the single-particle problem followed by a configuration-interaction approach to the many-body problem. We find three types of allowed transitions that would be naively expected to be forbidden.(i) Transitions that are parity forbidden in simple effective mass models with infinite confining wells (e.g. $1 S-2 S, 1 P-2 P$ ) but are possible by finite band-offsets and orbital-mixing effects; (ii) light-hole-to-conduction transitions, enabled by the confinement of light-hole states; and (iii) transitions that show and enhanced intensity due to electron-hole configuration mixing with allowed transitions. We compare these predictions with results of 8 -band $\mathbf{k} \cdot \mathbf{p}$ calculations as well as recent spectroscopic data. Transitions in (i) and (ii) explain recently observed satellites of the allowed $P-P$ transitions.
\end{abstract}

\section{INTRODUCTION}

In quantum dot spectroscopy, rather simple, idealized theoretical approaches have been applied to discuss which confined interband optical transitions are formally allowed and which are formally forbidden. But one expects the simple rules not to work. Yet, the mechanisms for failure have only been assessed within extensions of the simple models. To understand these mechanisms demands a high-level approach that naturally includes the complexity of the dots. Such approaches to the calculation of the optical properties are rare, with 8 -band $\mathbf{k} \cdot \mathbf{p}$ calculations being among the most sophisticate approaches used so far. Here, we discuss the nature of confined transitions in lensshaped (In, Ga)As/GaAs quantum dots by using an atomistic pseudopotential-based approach ${ }^{7.8 .9}$ Specifically, we study three mechanisms that render nominally forbidden transitions, in lower approximations, to allowed transitions within more realistic approximations: (i) " $2 S$-to$1 S$ " and " $2 P$-to- $1 P$ " "crossed" transitions allowed by finite band-offset effects and orbital mixing; (ii) transitions involving mixed heavy-hole and light-hole states, enabled by the confinement of light-hole states; and (iii) many-body configuration-mixing intensity enhancement enabled by electron-hole Coulomb interaction. We also compare our results with those of 8 -band $\mathbf{k} \cdot \mathbf{p}$ calculations. Our atomistic pseudopotential theory explains recent spectroscopic data.

\section{INTERBAND OPTICAL SPECTRUM OF (IN,GA)AS/GAAS DOTS}

\section{A. Method of calculation}

In our approach, the atomistic single-particle energies $\mathcal{E}_{i}$ and wave functions $\psi_{i}$ are solutions to the atomistic Schrödinger equation 7

$$
\left\{-\frac{1}{2} \nabla^{2}+V_{S O}+\sum_{l, \alpha} v_{\alpha}\left(\mathbf{r}-\mathbf{R}_{l, \alpha}\right)\right\} \psi_{i}=\mathcal{E}_{i} \psi_{i}
$$

where $v_{\alpha}$ is a pseudopotential for atom of type $\alpha$, with $l$-th site position $\mathbf{R}_{l, \alpha}$ in either the dot or the GaAs matrix. These positions are relaxed by minimizing the total elastic energy consisting of bond-bending plus bondstretching terms via a valence force field functional. $\frac{9}{-}$ This results in a realistic strain profile $\tilde{\varepsilon}(\mathbf{R})$ in the nanostructure ${ }^{10}$ In addition, $v_{\alpha}$ depends explicitly on the isotropic component of the strain $\operatorname{Tr}[\tilde{\varepsilon}(\mathbf{R})]{ }^{11} V_{S O}$ is a non-local (pseudo) potential that accounts for spinorbit coupling. $\frac{9}{-}$ In the single-particle approximation, the transition intensity for light polarized along $\hat{\mathbf{e}}$ is

$$
\left.I^{(S P)}(\omega ; \hat{\mathbf{e}})=\sum_{i, j}\left|\left\langle\psi_{i}^{(e)}|\hat{\mathbf{e}} \cdot \mathbf{p}| \psi_{j}^{(h)}\right\rangle\right|^{2}\right] \delta\left[\hbar \omega-\mathcal{E}_{i}^{(e)}+\mathcal{E}_{j}^{(h)}\right]
$$

where $\mathbf{p}$ is the electron momentum. 12

In addition to the single-particle effects, many-particle effects cause each of the monoexciton states $\Psi^{(\nu)}\left(X^{0}\right)$ to be a mixture of several electron-hole pair configurations (Slater determinants) $e_{i} h_{j}$. Namely,

$$
\left|\Psi^{(\nu)}\left(X^{0}\right)\right\rangle=\sum_{i, j} C_{i, j}^{(\nu)}\left|e_{i} h_{j}\right\rangle
$$

The coefficients $C_{i, j}^{(\nu)}$ are determined by the degree of configuration mixing allowed by the electron-hole Coulomb and exchange interaction 13 This mixing is determined by the symmetry of the $e-h$ orbitals and by their singleparticle energy separation. The many-body optical absorption for (incoherent) unpolarized light ${ }^{14}$ is given by

$$
I^{(M P)}(\hbar \omega)=\frac{1}{3} \sum_{\nu} \sum_{\hat{\mathbf{e}}=\hat{\mathbf{x}}, \hat{\mathbf{y}}, \hat{\mathbf{z}}}|M(\hat{\mathbf{e}})|^{2} \delta\left[\hbar \omega-E^{(\nu)}\left(X^{0}\right)\right],
$$




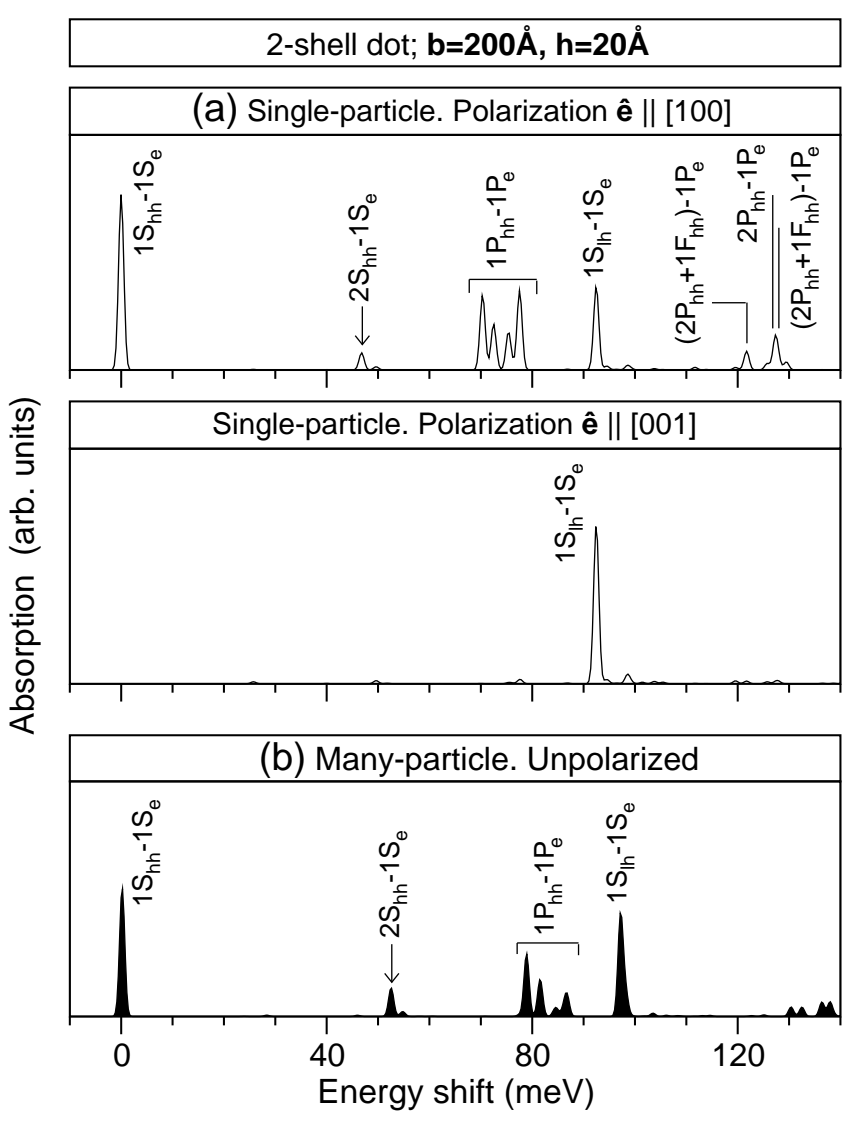

FIG. 1: Optical absorption spectrum of $X^{0}$ in a lens-shaped $\mathrm{In}_{0.6} \mathrm{Ga}_{0.4} \mathrm{As} / \mathrm{GaAs}$ quantum dot (base diameter $b=200 \AA$, height $h=20 \AA$ ) calculated at the (a) single-particle approximation [Eq. (2)] under in-plane (ê || [100]; top) and out-ofplane (री $\|$ [001]; bottom) polarization; and (b) at the manyparticle level [Eq. (4)] for unpolarized light. Energy is shown relative to (a) the single-particle gap $\mathcal{E}_{0}^{(e)}-\mathcal{E}_{0}^{(h)}=1333 \mathrm{meV}$ and (b) the ground-state energy $E^{(0)}\left(X^{0}\right)=1309 \mathrm{meV}$ of $X^{0}$.

where $M(\hat{\mathbf{e}})=\left\langle\Psi^{(\nu)}\left(X^{0}\right)|\hat{\mathbf{e}} \cdot \mathbf{p}| 0\right\rangle$. Thus, the configuration mixing can make transitions that are forbidden in the single-particle single-band approximation become allowed in the many-particle representation of Eq. (4) by borrowing oscillator strength from bright transitions.

\section{B. Results}

Figure 1 shows our calculated single-particle [Eq. (2); Fig. 1(a)] and many-particle [Eq. (4); Fig. [1(b)] absorption spectrum of $X^{0}$ for a lens-shaped $\mathrm{In}_{0.6} \mathrm{Ga}_{0.4} \mathrm{As} / \mathrm{GaAs}$ quantum dot with base diameter $b=200 \AA$ and height $h=20 \AA$ that confines two shells of electron states: $\left\{1 S_{e} ; 1 P_{e}\right\}$. The energy of the transitions is shown as a shift $\Delta \mathcal{E}$ from the singleparticle exciton gap $\mathcal{E}_{0}^{(e)}-\mathcal{E}_{0}^{(h)}$ [in Fig. [1(a)] or the ground-state energy of the monoexciton $E^{(0)}\left(X^{0}\right)$ [in Fig. [1 b)]. Figure 2 shows equivalent results for two dots with $b=252 \AA$ and heights $h=20 \AA$ and $35 \AA$, which

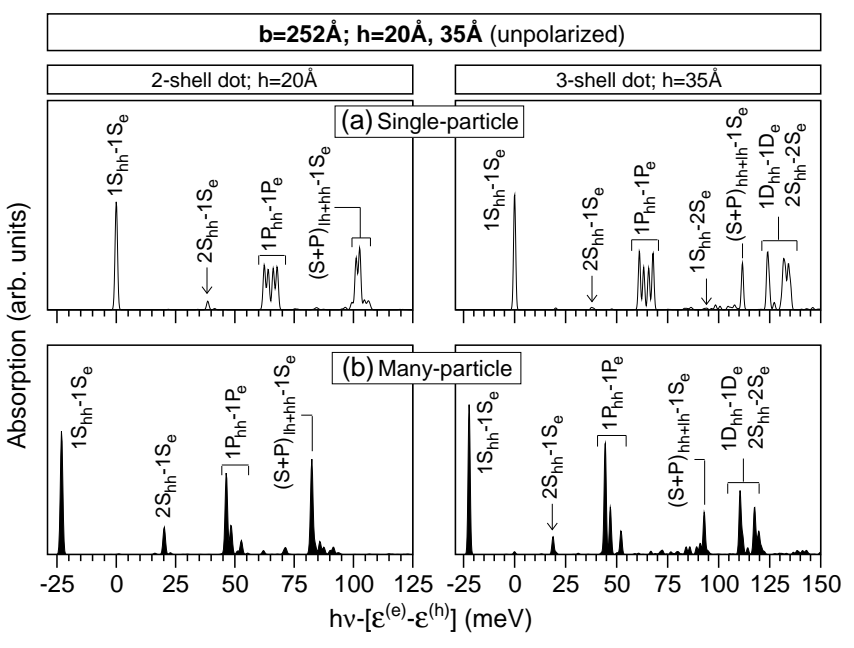

FIG. 2: Idem Fig. 1(b) for two $\operatorname{In}_{0.6} \mathrm{Ga}_{0.4} \mathrm{As} / \mathrm{GaAs}$ quantum dots that confine two (left panel) and three (right) shells of electron states, with heights $h=20 \AA$ and $30 \AA$, respectively, and base $b=252 \AA$.

confine two $\left\{1 S_{e} ; 1 P_{e}\right\}$ and three $\left\{1 S_{e} ; 1 P_{e} ; 1 D_{e}+2 S_{e}\right\}$ shells of electron states,respectively 15 As expected, we find nominally-allowed single-particle transitions, including (i) the fundamental transition $1 S_{h h}-1 S_{e}$ at $\Delta \mathcal{E}=$ $0 \mathrm{meV}$; (ii) the $1 P_{h h}-1 P_{e}$ transitions with energy shifts $\Delta \mathcal{E} \sim 75 \mathrm{meV}$ and $65 \mathrm{meV}$ for dots with $b=200 \AA$ and $252 \AA$, respectively; and (iii) the transitions $1 D_{h h^{-}}$ $1 D_{e}$ and $2 S_{h h}-2 S_{e}$ at $\Delta \mathcal{E} \sim 130 \mathrm{meV}$ for the three-shell $\operatorname{dot}(b=252 \AA, h=35 \AA)$. Note that the underlying atomistic $C_{2 v}$ symmetry of the circular-base lens-shape dot splits the electron and hole $1 P$ and $1 D$ states into 3 and 5 levels, respectively, and causes these states to be a mixture of $L_{z}= \pm 1$ and $L_{z}= \pm 2$, respectively. $\left[L_{z}\right.$ is the projection of the angular momentum along the cylindrical ([001], out-of-plane) axis of the dot.] Thus, in contrast to predictions of simplified models that assume $C_{\infty v}$ shape symmetry, transitions involving states $1 P$ and $1 D$ are split into four and nine lines, respectively (Figs. 1)and 21). We next discuss the nominally-forbidden transitions.

\section{Band-offset and orbital-mixing induced $1 S$-2S transitions}

If the electron and hole envelope wave functions are identical, the envelope-function selection rules indicate that only $\Delta i=0(i \rightarrow i)$ transitions are allowed, as assumed e.g. in Refs. 16 17 18 20. This will be the case in the single-band effective mass approximation if the confinement potential (band offset between dot and environment) for electron and hole are infinite. In contrast, we find a few $\Delta i \neq 0$ transitions with significant intensity: (i) $2 S_{h h}-1 S_{e}$ [Figs. 1(a) and 2(a)], which we find below $1 P_{h h}-1 P_{e}$; (ii) four transitions that involve the electron states $1 P_{e}$ and hole states $2 P_{h h}$ (also found in Ref. 21) and $2 P_{h h}+1 F_{h h}$ [Fig. 1(a)]; and 
(iii) transitions $1 S_{h h^{-}}-2 S_{e}$, and $2 S_{h h}-1 D_{e}$ and $1 D_{h h^{-}}-2 S_{e}$ in the three-shell dot (Fig. 21). There are two reasons why $\Delta i \neq 0$ transitions are allowed. First, in the case of finite band offsets or, equivalently, when the electron and hole wavefunctions are not identical, the condition $\Delta i=0$ is relaxed and transitions $j \rightarrow i$ may be allowed even in the effective-mass approximation. The latter happens to be the case in the work of Vasanelli et al. (Ref. 21) in which $2 S_{h h}-1 S_{e}$ and $2 P_{h h}-1 P_{e}$ transitions between confined electron and hole levels were found to have finite, non-negligible oscillator strength. Second, orbital mixing also makes such transition allowed: For example, a dot made of zinc-blende material and having a lens or cylindrical shape has the atomistic symmetry $C_{2 v}$ while spherical dots have $T_{d}$ symmetry. In contrast, continuum-like effective-mass based theories for dots use artificially higher symmetries. In fact, the ability of the envelope-function approximation to recognize the correct point-group symmetry depends on the number $\mathrm{N}$ of $\Gamma$ like bands used in the expansion. ${ }^{22} \mathrm{~N}=1$ corresponds to the "particle-in-a-box" or to the parabolic single-band effective mass approximation; $\mathrm{N}=6$ corresponds to including the valence band maximum (VBM) states only; and $\mathrm{N}=8$ corresponds to considering the VBM states plus the conduction band minimum. Higher values of $\mathrm{N}$ have been also considered ${ }^{23.24}$ In particular, (a) within the $\mathrm{N}=1$ single-band effective-mass approximation one uses the symmetry of the macroscopic shape (lens, cylinder, pyramid, sphere) rather than the true atomistic symmetry. For example, for zinc-blende lenses and cylinders one uses $C_{\infty v}$ symmetry rather than the correct $C_{2 v}$. (b) The 8 -band $\mathbf{k} \cdot \mathbf{p}$ Hamiltonian assumes cubic $\left(O_{h}\right)$ symmetry to describe the electronic structure of the dots, 25 the resulting symmetry group is dictated by both the symmetry of the macroscopic shape and the cubic symmetry. In the case of square-pyramid-shaped dot the symmetry is $C_{4 v}$ rather than the correct $C_{2 v}{ }^{26}$

In single-band effective-mass approaches, transition $i$ - $j$ is allowed as long as the overlap between the respective envelope functions is non-zero. For example, for spherical quantum dots one expects $S$ - $S$ transitions to be allowed but not $D-S$ transitions. Yet, in the true point group symmetry of the zinc-blende sphere the highest occupied hole state has mixed $S+D$ symmetry, which renders transition $1 S_{h}-1 D_{e}$ allowed, 27.28 and similarly the " $2 S-1 S$ " transition is allowed because the $2 S_{h h}$ state also contains $1 S_{h h}$ character ${ }^{29}$

\section{Strong light-hole-electron transitions}

In bulk zinc-blende semiconductors the valence-band maximum is made of degenerate heavy-hole ( $h h$, $|3 / 2, \pm 3 / 2\rangle)$ and light-hole $(l h,|3 / 2, \pm 1 / 2\rangle)$ states 30 While both optical transitions $h h-\Gamma_{1 c}$ and $l h-\Gamma_{1 c}$ are polarized in the $\hat{\mathbf{x}}-\hat{\mathbf{y}}$ plane, only the latter transition presents polarization along $\hat{\mathbf{z}}(\|[001])$. Under biaxial strain these $h h$ and $l h$ states split. In bulk, the rela-

(a) Strain-modified valence-band offsets

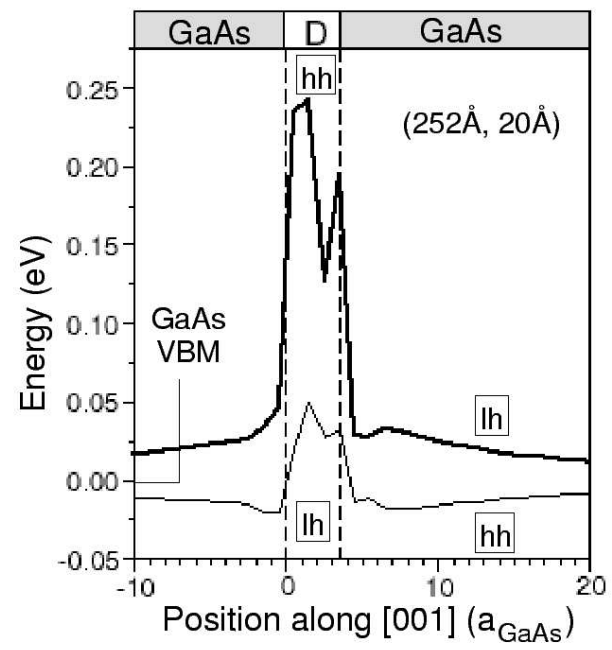

(b) 1st hole states with significant light-hole character

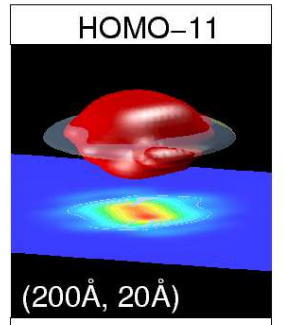

$22 \% \mathrm{hh} / 71 \% \mathrm{lh}$ $45.2 \mathrm{meV}$

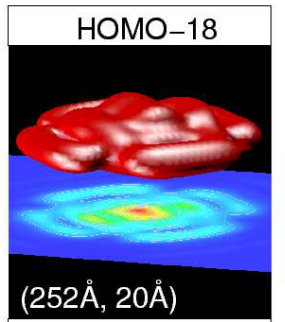

$43 \% \mathrm{hh} / 51 \% \mathrm{lh}$ 46.3meV

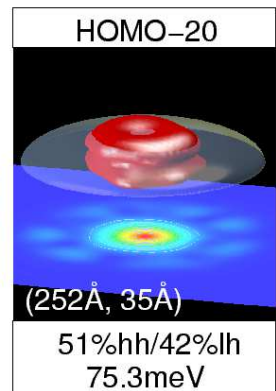

FIG. 3: (Color) (a) First (thick line) and second (thin line) strain-modified valence-band offsets along a line parallel [001] that pierces a lens-shaped $\mathrm{In}_{0.6} \mathrm{Ga}_{0.4} \mathrm{As} / \mathrm{GaAs}$ quantum dot through its center. The dot size is $b=252 \AA$ and $h=20 \AA$. Position is measured in units of the lattice parameter of GaAs $\left(a_{\mathrm{GaAs}}\right)$ and the energies are relative to the GaAs VBM $\left[E_{v}(\mathrm{GaAs})=-5.620 \mathrm{eV}\right]$. (b) Wave functions of the first hole state with significant $l h$ character for different $\operatorname{In}_{0.6} \mathrm{Ga}_{0.4} \mathrm{As} / \mathrm{GaAs}$ dots. Isosurfaces enclose $75 \%$ of the charge density, while contours are taken at $1 \mathrm{~nm}$ above the base. The energy $\mathcal{E}_{j}^{(h)}-E_{v}(\mathrm{GaAs})$ of the state appears in each panel.

tive energy of these states and their splitting depend on the strain: for compressive (e.g. InAs on GaAs) the $l h$ is below the $h h$ while for tensile (e.g. GaAs on InAs) the $l h$ is above the $h h .^{31}$ In quantum dots the energy of lh states is unknown. More importantly, it is generally assumed to be unconfined; so, the $l h-\Gamma_{1 c}$ transition is expected to be absent from spectroscopic data. Nonetheless, Minnaert et $a^{132}$ have speculated that despite the compressive strain in InAs/GaAs dots the $l h$ state is above the $h h$ states, while Ribeiro et $a^{i 33}$ have suggested the presence of a $l h$-derived state below the $h h$ states by measuring photo-reflectance and photo-absorption in (In,Ga)As/GaAs dots. Adler et $a^{134}$ and Akimov et a $a^{355}$ have also suggested the presence of $l h$-derived transi- 
tions in photoluminescence excitation (PLE) experiments in InAs/GaAs and CdSe/ZnSe self-assembled quantum dots, respectively. In addition, based on a 6 -band $\mathbf{k} \cdot \mathbf{p}$ calculation, Tadić et a $a^{136}$ have predicted that in diskshaped $\mathrm{InP} / \mathrm{In}_{0.51} \mathrm{Ga}_{0.49} \mathrm{P}$ dots the light-hole states are confined at the interface of the disk and become higher in energy than heavy-hole states as the thickness of the disk is increased.

We show in Fig. 3(a) the strain-modified valenceband offsets of a lens-shaped $\mathrm{In}_{0.6} \mathrm{Ga}_{0.4} \mathrm{As} / \mathrm{GaAs}$ with $b=252 \AA$ and $h=20 \AA$, calculated along a line normal to the dot base that pierces the dot through its center. The energy is presented relative to the GaAs VBM $\left(E_{v}(G a A s)=-5.620 \mathrm{eV}\right)$. We find that inside the dot the heavy-hole $(h h)$ potential is above the light-hole $(l h)$ one, while outside this order is reversed; although the $l h$ character of the lower-energy band-offset leaks slightly into the barrier close to the dot. Because the dot is alloyed the band offsets inside the dot are jagged. In agreement with Ribeiro et al, 33 but in contrast with Minnaert et $a l, 32$ our atomistic pseudopotential calculations reveal weakly confined light-hole states at energies deeper than the first $h h$ state. The wave functions of the first of these states are shown in Fig. 3(b). The energy spacing between the highest hole state [HOMO $\left.\left(\psi_{0}^{(h)}\right)\right]$ and the deep $l h$-type states increases from $92.4 \mathrm{meV}$ to $101.1 \mathrm{meV}$ and $111.7 \mathrm{meV}$ for HOMO-11 $\left[\psi_{11}^{(h)}\right]$, HOMO-18 $\left[\psi_{18}^{(h)}\right]$, and HOMO-20 $\left[\psi_{20}^{(h)}\right]$, respectively. These states give raise to two $l h$-derived transitions in the absorption spectra:

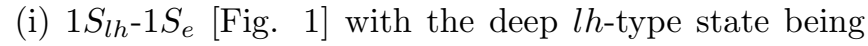
a mixture of $71 \% \mathrm{lh}$ and $22 \% \mathrm{hh}$. As seen in Fig. 1 this transition has a large intensity in both $\hat{\mathbf{e}} \|[100]$ and $\hat{\mathbf{e}} \|$ [001] polarizations. (ii) $(S+P)_{l h+h h^{-1}} S_{e}$ (Fig. 2) with $h h / l h$ percentages of $43 / 51$ and $51 / 42$ for the two-shell and three-shell dots, respectively. In these dots with $b=252 \AA$, the larger base size reduces the spacing between confined hole states and promotes character mixing. In the 2-shell dot, the offset energy of this transition with respect to $1 P_{h h}-1 P_{e}$ is $36.0 \mathrm{meV}$, in excellent agreement with the observed value 37 Note that simple models that follow the common assumption of unconfined $l h$ states do not explain the observed feature.

\section{Coulomb-induced transitions that are forbidden in the single-particle description}

Due to the electron-hole Coulomb interaction, each monoexciton state $\Psi\left(X^{0}\right)$ is a mixture of electron-hole configurations [Eq. (3)]. This mix results in a enhancement/diminishment of the intensity of both allowed and nominally-forbidden transitions in the absorption spectra [Fig. 2(b)]. These are shown by comparing Fig. 1(a) vs Fig. 1(b) and Fig. 2(a) vs Fig. 2(b). The many-body effects include (i) enhancement of the intensity of the nominally forbidden transition $2 S_{h h}-1 S_{e}$ particularly in the 3 -shell dot (Fig. 2); (ii) redistribution of the intensity of

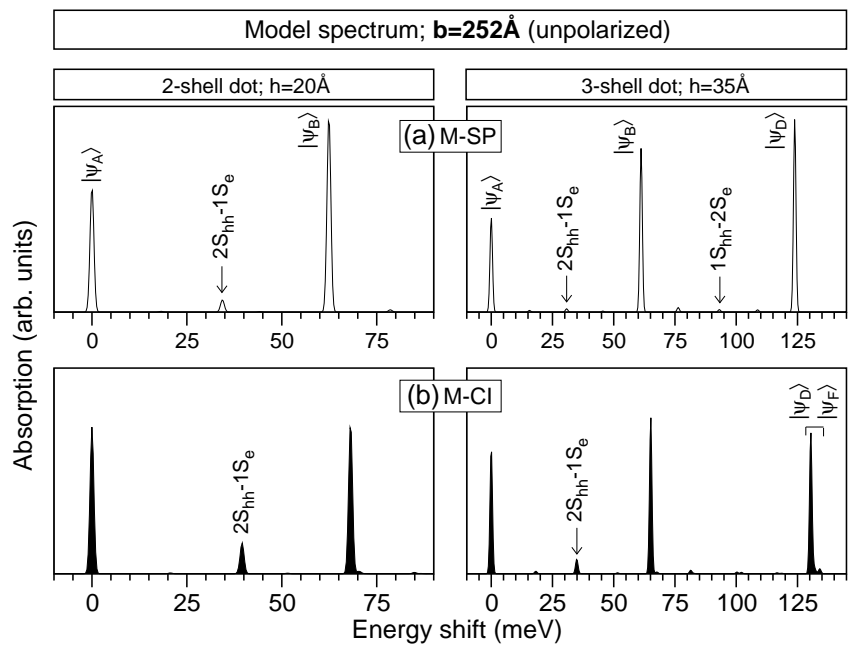

FIG. 4: Optical absorption spectrum of $X^{0}$ in two lensshaped $\operatorname{In}_{0.6} \mathrm{Ga}_{0.4} \mathrm{As} / \mathrm{GaAs}$ quantum dots that confine two (left panel) and three (right) shells of electron states. In both cases, the spectra are calculated within a model single-particle (M-SP) [Eq. (2)] (a) and configuration-interaction (M-CI) approach [Eq. (4)] (b), assuming degenerate $1 P$, and $2 D$ and $2 S$ (in three-shell dot) electron and hole states but retaining the atomistic wavefunctions. Vertical scales are different for each dot.

both the nominally allowed $1 P_{h h}-1 P_{e}$ transitions and the $1 D_{h h}-1 D_{e}$ and $2 S_{h h}-2 S_{e}$ transitions; and (iii) change of the intensity of the transitions involving deep hole states with significant light-hole character. The mixing enhancement $\eta\left(2 S_{h h}-1 S_{e}\right)=I^{(C I)}\left(2 S_{h h}-1 S_{e}\right) / I\left(2 S_{h h}-\right.$ $\left.1 S_{e}\right)=3.2$ and $\eta\left(1 S_{h h}-1 S_{e}\right)=1.1$ for the 2 -shell dot, while $\eta\left(2 S_{h h}-1 S_{e}\right)=8.2$ and $\eta\left(1 S_{h h}-1 S_{e}\right)=1.3$ for the three shell dot. For both dots, the enhancement of transition $2 S_{h h}-1 S_{e}$ arises mainly from configuration mixing with the four configurations $\left|1 P_{h h} 1 P_{e}\right\rangle$. The degree of mixing is small, $\sim 2 \%$ for both dots, due to the large $(\sim 26 \mathrm{meV})$ energy splitting between these electron-hole configurations at the single-particle (non-interacting) level, yet sufficient to cause a sizeable enhancement of the intensity. We find that the larger $\eta\left(2 S_{h h}-1 S_{e}\right)$ for the 3 -shell dot arises from a larger mixing with configuration $\left|1 S_{h h} 1 S_{e}\right\rangle$.

Comparison with experiment: The calculated $2 S_{h h}-1 S_{e}$ transition is $\sim 26 \mathrm{meV}$ below the strongest $1 P_{h h}-1 P_{e}$ transition; in excellent agreement with those observed by Preisler et $a^{337}$ in magneto-photoluminescence, who found $25 \mathrm{meV}$, and in contrast to the effective-mass approximation results of Vasanelli et al, which place $2 S_{h h^{-}}$ $1 S_{e}$ above $1 P_{h h}-1 P_{e}$. The calculated $1 S_{l h}-1 S_{e}$ transition is $18.3 \mathrm{meV}$ below $1 P_{h h}-1 P_{e}$, in only rough agreement with the value of $35 \mathrm{meV}$ observed by Preisler et al . $^{37}$

The effect of configuration-mixing on the optical spectrum was previously discussed within the simplified single-band 2D-EMA parabolic model ${ }^{17.18}$ Such continuum theories assume macroscopic shapes that lead to significant degeneracies among the single-particle states 
of Eq. (11): $P$ states are twofold degenerate; $D$ states and $2 S$ are degenerate; and the $S-P$ and $P-D$ energy spacings are equal. As a result, there is an artificially strong many-body mixing in Eq. (3). The many-particle exciton states with allowed Coulomb mixing are:

$$
\begin{aligned}
& \left|\Psi_{A}\right\rangle=\left|1 S_{h h} 1 S_{e}\right\rangle \\
& \left|\Psi_{B}\right\rangle=\frac{1}{\sqrt{2}}\left(\left|1 P_{h h}^{(+)} 1 P_{e}^{(+)}\right\rangle+\left|1 P_{h h}^{(-)} 1 P_{e}^{(-)}\right\rangle\right) \\
& \left|\Psi_{H}\right\rangle=\frac{1}{\sqrt{2}}\left(\left|2 S_{h h} 1 S_{e}\right\rangle+\left|1 S_{h h} 2 S_{e}\right\rangle\right) \\
& \left|\Psi_{D}\right\rangle=\frac{1}{\sqrt{3}}\left(\left|1 D_{h h}^{(+)} 1 D_{e}^{(+)}\right\rangle+\left|1 D_{h h}^{(-)} 1 D_{e}^{(-)}\right\rangle+\left|2 S_{h h} 2 S_{e}\right\rangle\right) \\
& \left|\Psi_{F}\right\rangle=\frac{1}{\sqrt{6}}\left(\left|1 D_{h h}^{(+)} 1 D_{e}^{(+)}\right\rangle+\left|1 D_{h h}^{(-)} 1 D_{e}^{(-)}\right\rangle-2\left|2 S_{h h} 2 S_{e}\right\rangle\right)
\end{aligned}
$$

Here, the $( \pm)$ labels indicate $L_{z}= \pm 1$ and \pm 2 for the $P$ and $D$ states, respectively. The Coulomb interaction couples states $\left|\Psi_{B}\right\rangle$ and $\left|\Psi_{H}\right\rangle$. Thus the $P-P$ transition is split in two lines $\Psi_{a}^{(s, p)} \simeq\left|\Psi_{B}\right\rangle+\left|\Psi_{H}\right\rangle$ and $\Psi_{b}^{(s, p)} \simeq\left|\Psi_{B}\right\rangle-\left|\Psi_{H}\right\rangle$. States $\left|\Psi_{D}\right\rangle$ and $\left|\Psi_{F}\right\rangle$, which arise from nominally allowed electron-hole configurations, are also coupled; consequently, the $D-D$ transition splits in two lines $\Psi_{a}^{(d, d)} \simeq\left|\Psi_{D}\right\rangle+\left|\Psi_{F}\right\rangle$ and $\Psi_{b}^{(d, d)} \simeq\left|\Psi_{D}\right\rangle-\left|\Psi_{F}\right\rangle$. The mixing enhancement $\eta\left(\left|\psi_{H}\right\rangle\right)$ within this model is $\infty$ (because transitions $2 S_{h h}-1 S_{e}$ and $1 S_{h h}-2 S_{e}$ are forbidden at the single-particle level).

To compare our atomistic predictions with the model calculations, we deliberately neglect in the pseudopotential-based calculation the atomistic-induced splitting of the $1 P$, and $1 D$ and $2 S$ states (but preserve their atomistically calculated wavefunctions). We calculate the absorption spectrum at the single-particle level [Eq. (2)] and separately in the many-particle approximation [Eq. (4)] ].38.39 Figures 4(a) and 4(b) show, respectively, the atomistic model calculation of the singleparticle and many-particle absorption spectra. By com-

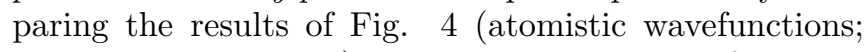
no $P$ or $D$ splittings) with the expectations from Eq. (55) (continuum wavefunctions; no $P$ or $D$ splittings), we find that (i) in the atomistic calculation the CI-enhanced transition corresponds to a mixture of states $\left|2 S_{h h} 1 S_{e}\right\rangle$ and $\left|\Psi_{B}\right\rangle$ instead of a mixture of $\left|\psi_{H}\right\rangle$ and $\left|\psi_{B}\right\rangle$ as in the model of Eq. (5); and (ii) the $D$-shell transition peak $\left[\left|\psi_{D}\right\rangle\right.$, Fig. [4(a)] splits in two transitions that correspond to a mixture of $\left|\psi_{D}\right\rangle$ and $\left|\psi_{F}\right\rangle$ as in the 2D-EMA model.

\section{Comparison with 8-band $\mathrm{k} \cdot \mathrm{p}$ calculations of the interband optical spectrum}

Other authors have calculated the absorption spectrum of pure, non-alloyed InAs/GaAs quantum dots using the 8-band $\mathbf{k} \cdot \mathbf{p}$ method with cubic symmetry. A comparison with our atomistic, pseudopotential-based predictions in alloyed (In,Ga)As/GaAs dots shows the following main features.

(i) Our prediction of transition $2 S_{h h}-1 S_{e}$ between $1 S-1 S$ and $1 P-1 P$ is consistent with the findings of nominally-forbidden transitions between $1 S$ - $1 S$ and $1 P$ $1 P$ by Heitz et $a l_{n}^{1}$, who calculated the (many-body) absorption spectrum of a monoexciton in pyramid-shaped non-alloyed InAs/GaAs dots with base length of $d=$ $170 \AA$ (height unspecified); Guffarth et al. ${ }^{2}$ who calculated the (many-body) absorption spectra of truncatedpyramid InAs/GaAs dots $(d=180 \AA, h=35 \AA)$; and the single-particle calculations of Sheng and Leburton ${ }^{3}$ in the case of a pure non-alloyed lens-shaped InAs/GaAs $\operatorname{dot}$ with $d=153 \AA$ and $h=34 \AA$. Conversely, other 8band $\mathbf{k} \cdot \mathbf{p}$ plus CI calculations did not predict nominallyforbidden transitions between $1 S-1 S$ and $1 P-1 P$, like those of Stier et $a l_{\underline{\underline{4}}}^{\underline{4}}$ for a truncated-pyramid InAs/GaAs dot with height $h=34 \AA$ (base lenght unspecified), who found three groups of transitions: $1 S-1 S, 1 P-1 P$, and $1 D-1 D$, without the presence of satellites around transitions $1 P-1 P$. Similarly, recent calculations by Heitz et $a l . \underline{5}$ of the absorption spectrum for small, flat $(d=136 \AA$ and heights from 3-7 ML) truncated-pyramid InAs/GaAs dots also predicted the absence of nominally-forbidden transitions between $1 S-1 S$ and $1 P-1 P$.

(ii) We predict that transitions $1 P-1 P$ and $1 D-1 D$ are split and span about $10 \mathrm{meV}$ and $15 \mathrm{meV}$ respectively. Instead, the $\mathbf{k} \cdot \mathbf{p}$-based calculations of Heitz et al $\mathbf{l}^{\underline{1}}$ predict that $1 P-1 P$ and $1 \mathrm{D}-1 \mathrm{D}$ transitions are much heavily split, each group spanning about $50 \mathrm{meV}$.

(iii) Sheng and Leburton ${ }^{6}$ calculated the singleparticle dipole oscillator strength for a truncatedpyramid InAs/GaAs dot with $d=174 \AA$ and $h=36 \AA$ and found strong nominally-forbidden transitions $1 D-1 P$ nearly $50 \mathrm{meV}$ above transitions $1 P-1 P$, and a transition HOMO-7-to-2 $S$. Our predictions differ from these in that we find transitions $\left(2 P_{h h}+1 F_{h h}\right)-1 P_{e}$ above $1 P-1 P$ [Fig. 1(a)]. In addition, in this energy interval $(\sim 50 \mathrm{meV}$ above $1 P-1 P$ ) we do not predict hole states with nodes along the [001] axis of the dots.

(iv) None of the 8-band $\mathbf{k} \cdot \mathbf{p}$-based plus CI calculations of Refs. 1 2 4 |5 predicted strong light-hole-toconduction transitions originating from deep, weaklyconfined hole states with predominant $l h$ character lying between the $1 P-1 P$ and $1 D-1 D$ transitions.

\section{CONCLUSION}

Atomistic, pseudopotential-based calculations of the excitonic absorption of lens-shaped (In, Ga)As/GaAs quantum dots predict nontrivial spectra that show nominally-forbidden transitions allowed by singleparticle band-offset effects as well as enhanced by manybody effects, and transitions involving deep, weakly confined hole states with significant light-hole character. These transitions explain the satellites of the $P-P$ nominally-allowed transitions recently observed in PLE. 


\section{Acknowledgments}

The authors thank G. Bester and L. He for valuable discussions, and V. Preisler (ENS, Paris) for making data in Ref. 37 available to them prior to publication. This work was funded by U.S. DOE-SC-BES-DMS, under Contract No. DE-AC3699GO10337 to NREL.
1 R. Heitz, O. Stier, I. Mukhametzhanov, A. Madhukar, and D. Bimberg, Phys. Rev. B 62, 11017 (2000).

2 F. Guffarth, R. Heitz, A. Schliwa, O. Stier, M. Geller, C. M. A. Kapteyn, R. Sellin, and D. Bimberg, Phys. Rev. B 67, 235304 (2003).

3 W. Sheng and J.-P. Leburton, phys. stat. sol. (b) 237, 394 (2003).

4 O. Stier, R. Heitz, A. Schliwa, and D. Bimberg, phys. stat. sol. (a) 190, 477 (2002).

${ }^{5}$ R. Heitz, F. Guffarth, K. Pötschke, A. Schliwa, D. Bimberg, N. D. Zakharov, and P. Werner, Phys. Rev. B 71, 045325 (2005).

6 W. Sheng and J.-P. Leburton, Appl. Phys. Lett. 80, 2755 (2002).

7 A. Zunger, phys. stat. sol. (b) 224,727 (2001).

8 L.-W. Wang and A. Zunger, Phys. Rev. B 59, 15806 (1999).

9 A. J. Williamson, L. W. Wang, and A. Zunger, Phys. Rev. B 62, 12963 (2000).

10 C. Pryor, J. Kim, L. W. Wang, A. J. Williamson, and A. Zunger, J. Appl. Phys. 83, 2548 (1998).

11 K. Kim, P. R. C. Kent, A. Zunger, and C. B. Geller, Phys. Rev. B 66, 045208 (2002) and references therein.

$12 \mathrm{P}$. Y. $\mathrm{Yu}$ and M. Cardona, Fundamentals of Semiconductors-Physics and Materials Properties (Springer, Berlin, 2003).

13 A. Franceschetti, H. Fu, L. W. Wang, and A. Zunger, Phys. Rev. B 60, 1819 (1999).

14 Unpolarized photons incoming from all directions.

15 G. A. Narvaez, G. Bester, and A. Zunger, J. Appl. Phys. 98, 043708 (2005).

16 U. Woggon, Optical properties of semiconductor quantum dots, (Springer-Verlag, Berlin, 1997).

17 G. A. Narvaez and P. Hawrylak, Phys. Rev. B 61, 13753 (2001).

18 P. Hawrylak, G. A. Narvaez, M. Bayer, and A. Forchel, Phys. Rev. Lett. 85, 389 (2000).

19 F. Findeis, A. Zrenner, G. Böhm, and G. Abstreiter, Solid State Comm. 114, 227 (2000).

20 U. Honester, R. Di Felice, E. Molinari, and F. Rossi, Appl. Phys. Lett. 75, 3449 (1999).

21 A. Vasanelli, R. Ferreira, and G. Bastard, Phys. Rev. Lett. 89, 216804 (2002).

22 A. Zunger, phys. stat. sol. (a) 190, 467 (2002).

${ }^{23}$ N. Rougemaille, H.-J. Drouhin, S. Richard, G. Fishman, and A. K. Schmid, Phys. Rev. Lett. 95, 186406 (2005); C. W. Cheah, L. S. Tan, G. Karunasiri, J. Appl. Phys. 91,
5105 (2002); W. H. Lau, J. T. Olesberg, and M. E. Flatté, Phys. Rev. B 64, 161301(R).

24 S. Richard, F. Aniel, and G. Fishman, Phys. Rev. B 70, 235204 (2004); Phys. Rev. B 71, 169901(E) (2005).

25 C. Pryor, Phys. Rev. B 57, 7190 (1998); W. Sheng and J.-P. Leburton, Phys. Stat. Sol. (b) 237, 394 (2003).

${ }^{26}$ It is possible to have an 8 -band $\mathbf{k} \cdot \mathbf{p}$ Hamiltonian for strained zinc-blende semiconductors with the correct tetrahedral $\left(T_{d}\right)$ symmetry. [See H.-R. Trebin, U. Rössler, and R. Ranvaud, Phys. Rev. B 20, 686 (1979).] If this Hamiltonian is used to find the electronic energy-level structure of a square-pyramid-shaped InAs/GaAs dot the symmetry of the problem would reduce to $C_{2 v}$.

27 J.-B. Xia, Phys. Rev. B 40, 8500 (1989).

${ }^{28}$ H. Fu and A. Zunger, Phys. Rev. B 56, 1496 (1997).

29 Band-mixing effects mix the $1 S$ and $2 S$ character of the single-particle hole states.

30 G. Bastard, Wave Mechanics Applied to Semiconductor Heterostructures (Halstead, New York, 1988).

31 P. R. C. Kent, G. L. W. Hart, and A. Zunger, Appl. Phys. Lett. 81, 4377 (2002).

32 A. W. E. Minnaert, A. Yu. Silov, W. van der Vleuten, J. E. M. Haverkort, and J. H. Wolter, Phys. Rev. B 63, 075303 (2001).

33 E. Ribeiro, F. Cerdeira, M. J. S. P. Brasil, T. Heinzel, K. Ensslin, G. Medeiros-Ribeiro, and P. M. Petroff, J. Appl. Phys. 87, 7994 (2000).

${ }^{34}$ F. Adler, M. Geiger, A. Bauknetch, F. Scholz, H. Schweizer, M. H. Pilkuhn, B. Ohnesorge, and A. Forchel, J. Appl. Phys. 80, 4019 (1996).

35 A. I. Akimov, A. Hundt, T. Flissikowski, P. Kratzert and F. Henneberger, Physica E 17, 31 (2003); T. Flissikowski, I. A. Akimov, A. Hundt, and F. Henneberger, Phys. Rev. B 68, 161309(R) (2003).

36 M. Tadić, F. M. Peeters, and K. L. Janssens, Phys. Rev. B 65, 165333 (2002).

37 V. Preisler, T. Grange, R. Ferreira, L. A. de Vaulchier, Y. Guldner, F. J. Teran, M. Potemski, and A. Lemaitre, Phys. Rev. B 73, 075320 (2006).

38 Both dots confine more than three shells of hole states but we only consider three in this model calculation.

${ }^{39}$ In the pseudopotential-based plus CI calculation we do not use Eq. (5), but we let the Coulomb interaction mix the configurations $\left|e_{i} h_{j}\right\rangle$ that compose the monoexciton states [Eq. [3] ]. We include the electron-hole direct Coulomb coupling but neglect the much smaller electron-hole exchange. 